\title{
Caries dental en escolares de 6 a 12 años de Navolato, Sinaloa: resultados preliminares.
}

Carta al Editor

Juan J. Villalobos-Rodelo¹, Carlo E. Medina-Solís², Ana A. Vallejos-Sánchez³ , José L. Espinoza-Beltrán.

${ }^{1}$ Maestría en Salud Pública del Instituto Nacional de Salud Pública. Práctica Privada. Navolato, Sinaloa. ${ }^{2}$ Centro de Investigación en Sistemas de Salud del Instituto Nacional de Salud Pública, Cuernavaca, Morelos, México. ${ }^{3}$ Facultad de Odontología de la Universidad Autónoma de Campeche, Campeche, México, ${ }^{4}$ Subdireccion de Salud Bucal de los Servicios de Salud de Sinaloa, Culiacán, Sinaloa, México.

La caries dental es un proceso que implica un desequilibrio de las interacciones moleculares normales entre la superficie/subsuperficie del diente y el biofilm microbiano adyacente. Este desequilibrio se manifiesta en un cierto plazo como desmineralización acumulativa del diente que, si es desenfrenado, tiene el potencial de producir cavitación del esmalte y daño colateral a la dentina y a la pulpa, culminando con la destrucción localizada de los tejidos duros del diente (1). Se considera que la severidad y el costo social de la caries dental en niños de edad preescolar y escolar, son considerables. Diversos autores (2-7) han indicado que es un problema de salud pública debido a su alta prevalencia e incidencia, además tiene la característica de que se encuentra concentrada en poblaciones con desventajas sociales en México y en el Mundo.

Según el US Department of Health and Human Services, la caries dental es la enfermedad infantil crónica más común en Estados Unidos (6). Como ha sido señalado por algunos autores (8), todavía son escasos los estudios en el campo de la salud bucal en
México. Contamos con muy poca información sobre la magnitud de los principales problemas bucales.

En la ciudad de Navolato, Sinaloa, realizamos un estudio transversal en 3048 niños de 6 a 12 años de edad, asistentes a 18 escuelas primarias con el objetivo de determinar la experiencia (promedio de dientes temporales/permanentes cariados, extraídos/ perdidos y obturados), prevalencia (porcentaje de niños con dientes temporales/permanentes cariados, extraídos/perdidos y obturados [ceod/CPOD > 0] $[\mathrm{c}=$ dientes temporales con caries evidente, $\mathrm{e}=$ dientes temporales con extracción indicada por caries, $\mathrm{o}=$ dientes temporales obturados y que no presentan lesión cariosa, $\mathrm{d}=$ unidad diente; $\mathrm{C}=$ dientes permanentes con caries evidente, $\mathrm{P}=$ dientes permanentes perdidos por caries, $\mathrm{O}=$ dientes permanentes obturados y que no presentan lesión cariosa, $\mathrm{D}=$ unidad diente]) y severidad de caries (ceod/CPOD $\geq 3$ y ceod/CPOD $\geq 6$ ) en la dentición temporal y permanente. Los dientes permanentes de 3018 niños y la dentición temporal de 2629 fueron

Solicitud de sobretiros: M en C. Carlo E. Medina-Solís. Privada de Altillo S/N entre Av. Central y Pedro Moreno. Colonia San José,

C.P. 24040. Campeche, Campeche, México.

Tel: + (981)81 10215, Correo electrónico: cemedinas@yahoo.com

Recibido el 2/Junio/2005. Aceptado para publicación el 9/Septiembre/2005.

Este artículo está disponible en http://www.uady.mx/sitios/biomedic/revbiomed/pdf/rb0516310.pdf

Vol. 16/No. 3/Julio-Septiembre, 2005 
JJ Villalobos-Rodelo, CE Medina-Solís, AA Vallejos-Sánchez, JL Espinoza-Beltrán.

evaluados. Treinta sujetos presentaron sólo dentición temporal, 419 sólo dentición permanente y 2599 tuvieron dentición mixta. Todos los sujetos fueron examinados clínicamente por uno de dos examinadores capacitados y con entrenamiento estandarizado (Kappa $>0.85)$ en el criterio para detectar caries dental. Se utilizó para medir las variables clínicas un espejo bucal plano y luz natural, de acuerdo a los criterios establecidos por la OMS. Se calcularon los índices comúnmente utilizados en estudios sobre caries dental, así como el nuevo índice caries significante ( $\mathrm{SiC})$. Para el análisis bivariado se utilizaron pruebas de $\div^{\circ}$, U de Mann-Whitney, Kruskall-Wallis y de tendencias no-paramétrica, según la escala de medición de las variables. Los análisis se realizaron en STATA 8.2. El promedio de los índices ceod y CPOD fue de $4.68 \pm 3.21$ y 3.24 \pm 2.72 , respectivamente.

El CPOD para toda la muestra fue de $3.24 \pm$ 2.72 y el ceod de $4.68 \pm 3.21$. A los 12 años el CPOD fue de $6.49 \pm 3.85$. La proporción de niños libres de caries en ambas denticiones fue de 3.7\%. La prevalencia de caries en la dentición primaria y permanente fue de 90.0 y $82.0 \%$, respectivamente, $y$ en la dentición primaria para los de 6 años de edad fue de $95 \%$. El SiC fue de 5.91 para toda la población y 10.87 para los de 12 años de edad. En el cuadro 1 se presenta la distribución por grupos de edad de la prevalencia y severidad de caries dental. Las niñas estuvieron más afectadas por caries, tanto en experiencia como en prevalencia y severidad, que los niños $(\mathrm{p}<0.01)$ en su dentición permanente, no así en la dentición temporal $(\mathrm{p}>0.05)$. Se observaron diferencias significativas por edad $(\mathrm{p}<0.001)$ en la experiencia de caries, cuando realizamos la prueba de tendencias no paramétrica; observamos que el CPOD aumenta cuando lo hace la edad y el ceod disminuye cuando la edad se incrementa $(p<0.001)$. El componente "caries" de los índices ceod y CPOD contribuyó con el mayor porcentaje (83.3\% y 95.4\%, respectivamente) en su construcción.

En comparación con estudios $(5,9,10)$ previos realizados en México, nuestros resultados fueron desfavorables. El presente estudio confirmó lo observado en otras investigaciones (5, 9-11) con relación a la edad y a la experiencia de caries dental en la dentición permanente: a mayor edad, mayor presencia de caries. Esta relación ocurrió tanto en los

Cuadro 1

Distribución de caries dental por edad de acuerdo a los índices de caries ceod ${ }^{1}, \mathrm{CPOD}^{2}$, porcentaje de sujetos libres de caries $(\operatorname{ceod} / \mathrm{CPOD}=0$ ), baja severidad (ceod/CPOD $\geq 3)$ y alta severidad (ceod/CPOD $\geq 6)$ y el $\mathrm{SiC}$ en escolares de Navolato Sinaloa en 2004.

\begin{tabular}{|c|c|c|c|c|c|c|c|c|c|}
\hline Edad & $\bar{x} \pm \operatorname{ceod}(n)^{* \dagger}$ & $\bar{x} \pm \operatorname{CPOD}(n) * t$ & $\begin{array}{c}\% \\
\operatorname{ceod}=0\end{array}$ & $\begin{array}{c}\% \\
\text { CPOD=0 }\end{array}$ & $\begin{array}{c}\% \\
\operatorname{ceod} \geq 3\end{array}$ & $\begin{array}{c}\% \\
\text { CPOD } \geq 3\end{array}$ & $\begin{array}{c}\% \\
\operatorname{ceod} \geq 6\end{array}$ & $\begin{array}{c}\% \\
\text { CPOD } \geq 6\end{array}$ & $\mathrm{SiC}$ \\
\hline 6 & $6.41 \pm 3.65(380)$ & $1.04 \pm 1.33(357)$ & 5.0 & 50.4 & 78.2 & 8.7 & 46.1 & 0.0 & $2.65 \pm 1.02$ \\
\hline 7 & $5.73 \pm 3.31(461)$ & $1.70 \pm 1.59(454)$ & 7.4 & 34.4 & 74.6 & 21.4 & 40.6 & 0.2 & $3.68 \pm 0.65$ \\
\hline 8 & $5.55 \pm 2.98(503)$ & $2.48 \pm 1.63(504)$ & 5.4 & 18.9 & 75.2 & 38.3 & 36.6 & 0.4 & $4.15 \pm 0.55$ \\
\hline 9 & $4.28 \pm 2.76(530)$ & $3.16 \pm 1.79(554)$ & 10.2 & 10.5 & 59.1 & 53.8 & 22.3 & 2.7 & $4.78 \pm 1.23$ \\
\hline 10 & $3.36 \pm 2.32(426)$ & $3.94 \pm 2.28(509)$ & 13.2 & 6.5 & 45.2 & 65.0 & 9.6 & 10.2 & $6.16 \pm 2.03$ \\
\hline 11 & $2.25 \pm 2.00(253)$ & $5.29 \pm 3.21(434)$ & 20.6 & 3.5 & 23.7 & 74.4 & 4.3 & 27.2 & $8.89 \pm 2.69$ \\
\hline 12 & $2.00 \pm 1.94(70)$ & $6.49 \pm 3.85(206)$ & 22.9 & 2.4 & 15.5 & 82.0 & 4.2 & 39.8 & $10.87 \pm 3.01$ \\
\hline Total & $4.68 \pm 3.21(2623)$ & $3.24 \pm 2.72(3018)$ & 9.8 & 18.0 & 60.8 & 47.8 & 27.4 & 9.0 & $5.91 \pm 2.64$ \\
\hline
\end{tabular}

* Prueba de Kruskal-Wallis $p<0.001 ; †$ Prueba no-paramétrica para tendencias $=-21.06, p=0.000 ; \$$ Prueba noparamétrica para tendencias $=31.80, p=0.000$

1 ceod: $\mathrm{c}=$ dientes temporales con caries evidente, $\mathrm{e}=$ dientes temporales con extracción indicada por caries, $\mathrm{o}=$ dientes temporales obturados y que no presentan lesión cariosa, $d=$ unidad diente.

${ }^{2} \mathrm{CPOD}$ : $\mathrm{C}=$ dientes permanentes con caries evidente, $\mathrm{P}=$ dientes permanentes perdidos por caries, $\mathrm{O}=$ dientes permanentes obturados y que no presentan lesión cariosa, $\mathrm{D}=$ unidad diente. 


\section{Caries dental en escolares.}

porcentajes de niños libres de caries en cada grupo etáreo como en el $\mathrm{SiC}$. Es razonable pensar que la mayor edad permite un mayor tiempo de exposición al ambiente ácido bucal. Igualmente, es inevitable resaltar la baja experiencia de dientes restaurados ( $<20 \%$ de los índices ceod/CPOD), común en países en vías de desarrollo (5, 9-11).

Un primer paso para la planeación de los servicios de salud bucal es el conocimiento del perfil epidemiológico de la población. Así, basados en este conocimiento se pueden planear diferentes estrategias preventivas y curativas en estos niños escolares.

La necesidad de realizar estudios epidemiológicos, como forma de monitorear las condiciones de salud bucal en las poblaciones ha sido ampliamente recomendada por la OMS. En este sentido es necesario efectuar estudios que revelen la situación real de la población para llevar a cabo la planeación de programas de salud bucal que se adapten a las necesidades de tratamiento restaurador de la población.

A manera de conclusión podemos decir que se observaron altos índices de caries así como alta prevalencia y severidad de caries en ambas denticiones. Comparando nuestros resultados con las metas de salud bucal de la OMS, podemos decir que no se lograron los objetivos de la OMS propuestos para el año 2000. Por lo que es necesario implementar estrategias que mejoren las condiciones de salud bucal en estos niños y lograr las metas para el año 2015. Estas estrategias deben incluir, además del componente preventivo, un componente restaurativo.

Palabras clave: Caries dental, epidemiología, prevalencia, índice ceod, índice CPOD, escolares, Sinaloa.

\section{REFERENCIAS.}

1.- Pitts NB, Stamm JW. International Consensus Workshop on Caries Clinical Trials (ICW-CCT) - Final consensus statements: Agreeing where the evidence leads. J Dent Res 2004; 83 (Spec Iss C):C125-C128.

2.- Maupomé G, Borges SA, Ledesma C, Herrera R, Leyva ER, Navarro A. Prevalencia de caries en zonas rurales y periurbanas marginadas. Salud Pública Mex 1993; 35:35767.

3.- Maupomé G. An introspective qualitative report on dietary patterns and elevated levels of dental decay in a deprived urban population in northern Mexico. ASDC J Dent Child 1998;65:276-85.

4.- Irigoyen ME, Maupome G, Mejia AM. Caries experience and treatment needs in a 6- to 12-year-old urban population in relation to socio-economic status. Community Dent Health 1999; 16:245-9.

5.- Herrera MS, Medina-Solís CE, Rosado-Vila G, MinayaSánchez M, Vallejos-Sánchez AA, Casanova-Rosado JF. Prevalencia, severidad de caries y necesidades de tratamiento en preescolares de una comunidad suburbana de Campeche-2001. Bol Med Hosp Infant Mex 2003; 60:18996.

6.- US Department of Health and Human Services. Oral Health in America: A report of the Surgeon General. Rockville, MD: US Department of Health and Human Services, National Institutes of Dental and Craniofacial Research, National Institutes of Health; 2000.

7.- Pitts NB, Stamm JW. Proceeding clinical. Preface. J Dent Res 2004; 83 (Spec Iss C):C4-C5.

8.- Moreno-Altamirano A. Propone aplicar el índice comunitario de fluorosis (Carta al editor). Salud Pública Mex 2001;43:180-1.

9.- Casanova-Rosado AJ, Medina-Solís CE, CasanovaRosado JF, Vallejos-Sánchez AA, Maupomé G, Ávila-Burgos L. Dental caries and associated factor in Mexican schoolchildren aged 6-13 years. Acta Odontol Scand 2005; (en prensa).

10.- Juárez-López ML, Hernández-Guerrero JC, Jiménez-Farfán D, Ledesma-Montes C. Prevalencia de fluorosis dental y caries en escolares de la ciudad de México. Gac Med Mex 2003; 139:221-5.

11. Herrera M, Medina-Solís CE, Maupomé G. Experiencia y prevalencia de caries dental en escolares de León, Nicaragua. Gaceta Sanitaria 2005; (en prensa). 